\title{
Improving the Performance of Finger-Vein Recognition System Using A New Scheme of Modified Preprocessing Methods
}

\author{
${ }^{1}$ Ahmed A. Mustafa, ${ }^{2}$ Ahmed AK. Tahir \\ ${ }^{1,2}$ College of Science, Duhok University, Kurdistan Region, Iraq
}

\begin{abstract}
This paper aims at improving the performance of finger-vein recognition system using a new scheme of image preprocessing. The new scheme includes three major steps, RGB to Gray conversion, ROI extraction and alignment and ROI enhancement. ROI extraction and alignment includes four major steps. First, finger-vein boundaries are detected using two edge detection masks each of size $(4 \times 6)$. Second, the correction for finger rotation is done by calculating the finger base line from the midpoints between the upper and lower boundaries using least square method. Third, ROI is extracted by cropping a rectangle around the center of the finger-vein which is determined using the first and second invariant moments. Forth, the extracted ROI is normalized to a unified size of $192 \times 64$ in order to compensate for scale changes. ROI enhancement is done by applying the technique of Contrast-Limited Adaptive Histogram Equalization (CLAHE), followed by median and modified Gaussian high pass filters. The application of the given preprocessing scheme to a finger-vein recognition system revealed its efficiency when used with different methods of feature extractors and with different types of finger-vein database. For the University of Twente Finger Vascular Pattern (UTFVP) database, the achieved Identification Recognition Rates (IRR) for identification mode using three feature extraction methods Local Binary Pattern (LBP), Local Directional Pattern (LDP) and Local Line Binary Pattern (LLBP) are (99.79, 99.86 and 99.86) respectively, while the achieved Equal Error Rates (EER) for verification mode for the same feature extraction methods are $(0.139,0.069$ and 0.035$)$. For the Shandong University Machine Learning and Applications - Homologous Multi-modal Traits (SDUMLA-HMT) database, the achieved Identification Recognition Rates (IRR) for identification mode using three feature extraction methods LBP, LDP and LLBP are $(99.57,99.73$ and 99.65) respectively, while the achieved Equal Error Rates (EER) for verification mode for the same feature extraction methods are $(0.419,0.262$ and 0.341$)$. These results outrage those of the previous state-of-art methods.

KEY WORDS: Human Biometrics, Finger-vein recognition, Feature extraction, Preprocessing, Recognition Performance.
\end{abstract}

\section{Introduction}

Finger-vein recognition is relatively a recent biometric authentication system compared to other biometric recognition systems. It is used in various applications, such as e-commerce (e.g. online banking and ATM machines), different forms of security access control (e.g. border checking, door access control and building access), automobile security, employee time and attendance tracking, computer and network authentication and so on ${ }^{[1,2]}$.

The pattern of finger-vein based blood vessel is unique for each individual like other human biometrics such as the patterns of fingerprints, iris, palm vein, etc. Fingervein may be more secure and acceptable and at the same time less expensive for authentication compared to some of other biometrics. For instance, finger-vein provides more secure and safety compared to fingerprints because veins are located under the surface of the skin and will not be affected by skin damage and because they are living bodies so they cannot be cheated[3]. Besides, finger-vein may be more acceptable compared to iris even the later is known of lower error rates for authentication because some users show resistance to the direct application of light rays into their eyes ${ }^{[4]}$. Moreover, finger-veins are less affected by environment conditions such as sweaty, dry and slightly dirty fingers and weather conditions such as humidity, high and low temperature.

Finger-vein recognition systems typically consist of four main modules, finger-vein image acquisition, image preprocessing, feature extraction and finally feature 
matching. Image acquisition involves the portrayed of the finger-vein images by a device that is capable of providing good quality images under constant conditions of illumination and with least degree of finger misalignment. Image preprocessing involves the detection of the finger region, localization of the Region of Interest (ROI), ROI correction for rotation, ROI cropping, normalizing the ROI, and finally ROI enhancing. Feature extraction involves the extraction of the most unique features from the veins. Matching stage involves a comparison between the extracted features of the tested finger-veins with the already enrolled fingervein feature templates. Figure (1) shows the blockdiagram of a typical finger-vein recognition system with two modes, enrollment and recognition.

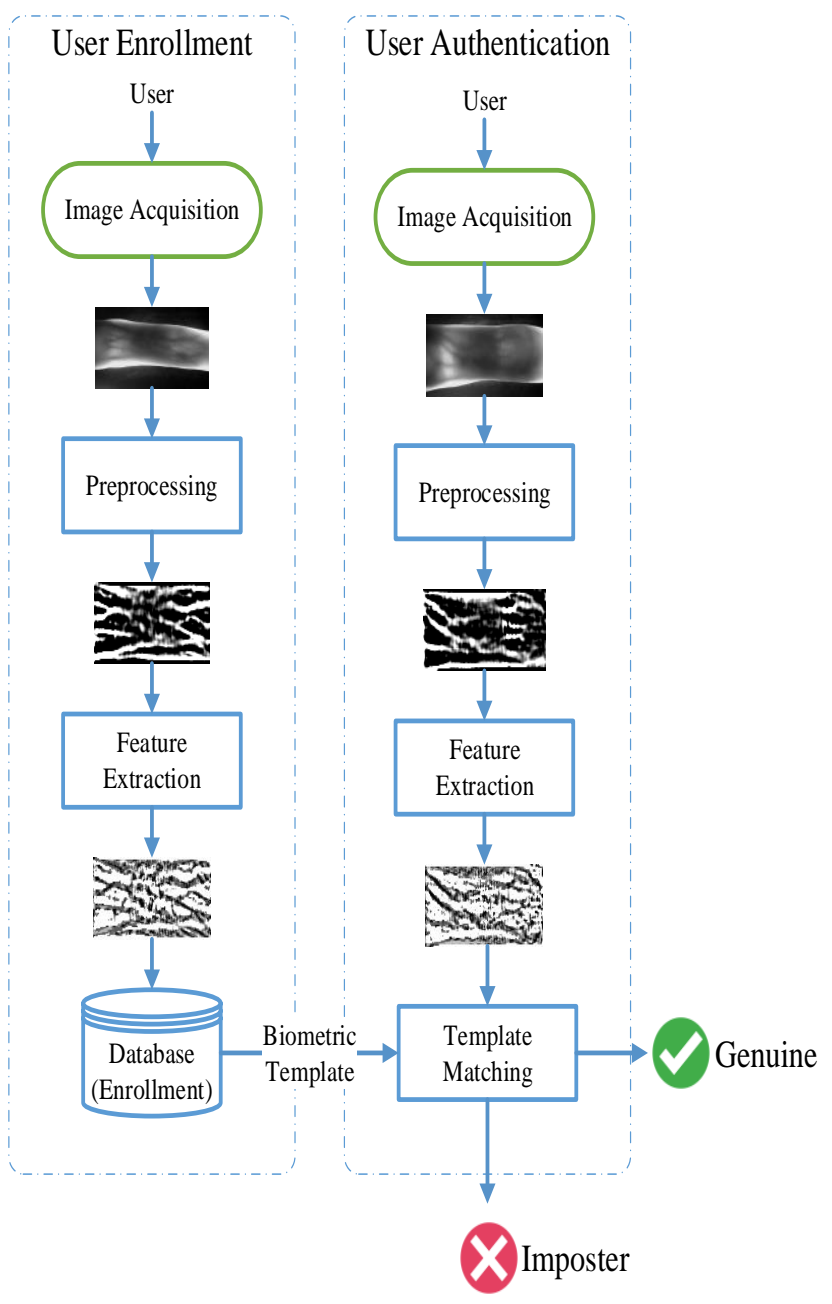

Fig. 1. The block-diagram of finger-vein recognition system

Image preprocessing is an important stage in finger-vein recognition system and without preprocessing the performance of the recognition may be degraded by the quality of the finger-vein images[5]. The main objectives of preprocessing are first to perform finger position alignment and second to improve the uneven and poor contrast of the image in order to be more ready for feature extraction[6].Unlike other biometrics, in fingervein recognition, the quality of the image can be poor due to a number of reasons like the existence of undesirable black background, transitional variation, rotational variation, and fluctuations of the NIR light. In addition, the image acquiring conditions and acquisition device performance may lead to a number of problems such as low contrast, noise and shades[7]. Thus for better performance, the acquired finger-vein images need to be aligned and enhanced prior to the feature extraction stage. In particular, the extraction of ROI is important to make the intra-set variance (within class variance) minimum and inter-set variance (between classes variance) as maximum as possible.

The objective of this paper is to introduce a new scheme of modified methods for finger-vein preprocessing that can be applied efficiently to different databases with different feature extractors.

The remainder of this paper is organized as follows. In Section 2, a review of the related work is given. In Section 3 , the proposed method of preprocessing is introduced and in sections (4-6) the details of the modified methods are given. In section 7 , a brief description of the used databases is presented. In section 8 , the results of applying the proposed scheme to University of Twente Finger Vascular Pattern (UTFVP) and Shandong University Machine Learning and Applications - 
Homologous Multi-modal Traits (SDUMLA-HMT)

databases are presented and compared with the results of previous works. Finally, the conclusions are given in section 9 .

\section{RELATED WORKS}

Lots of research works concerning the preprocessing of finger-vein images have been developed to improve the quality of finger-vein images to increase the accuracy of finger-vein recognition system. For instance, Lee and others ${ }^{[8]}$ suggested an image preprocessing scenario that implement finger-vein region localization, alignment and enhancement. They used two $(4 \times 20)$ masks, which were known later as Lee masks, for finger boundary detection and ROI extraction. For ROI alignment they used minutia points and affine transform to correct for scale, translation and rotation errors. However, their method seemed to be time consuming as the convolution with two large filter masks and the determination of minutia points from sampled images require somehow a long computation time. Park ${ }^{[9]}$ used the two Lee masks for finger boundaries detection, but he did the alignment manually on the device during image capturing to reduce the computation time, but in reality manual alignment by itself is a time consuming action and may have negative impacts during the operation of the system as user intervention means no more automatic system. Rosdi and others ${ }^{[10]}$ used image binarization followed by Otsu's threshold method to detecting the center of the finger for ROI extraction. Then a modified Gaussian high-pass filter is used for ROI enhancement and finally they used the Phase Only Correlation (POC) function ${ }^{[11]}$ for ROI alignment. Park and Park ${ }^{[12]}$ considered the direction and thickness of the vein line which were determined based on an optimal eight and four directional Gabor filters. This method again seemed to be time consuming because of the two steps use of Gabor filtering. Wang and others ${ }^{[5]}$ used image threshold to binarize the finger-vein image followed by ROI extraction. Pang and others ${ }^{[13]}$ used Sobel filter to outline the finger-vein boundaries then an inner rectangle was selected as ROI. For enhancement, histogram equalization was used. Peng and other [14] used two filter masks for finger region localization each of size $2 \times 2$ to speed up the process followed by Hough transform to recover the missing points on finger boundary. For alignment, they used an affine transform which was generated by calculating finger contour and the central line. For ROI segmentation, they used histogram projection technique. For ROI enhancement, they used a Non-Local Means (NLM) algorithm. However, the achieved Equal Error Rate (EER) was not promising and their method suffers from the complex computation drawback due to the use of Hough transform for fitting the missing points on the finger border and the implementation of NLM algorithm. Lu and others[15] Used open operation followed by Hough transform for ROI detection. They used Adaptive Wavelet Shrinkage (AWS) for denoising and the Contrast Limited Adaptive Histogram Equalization (CLAHE) for ROI enhancement. They achieved $98.79 \%$ recognition rate using SDUMLAHMT. In another study, Lu and others ${ }^{[16]}$ used a method of three steps, ROI localization using Hough transform, Denoising using multi-resolution analysis and ROI enhancement using CLAHE technique. They obtained IRR values of $(98.48,98.56$ and 99.21) with Local Binary Pattern (LBP), Local Line Binary Pattern (LLBP) and Polydirectional Local Line Binary Pattern (PLLBP) respectively using SDUMLA-HMT database. Xie and others ${ }^{[17]}$ applied Prewitt operator followed by image binarization then rotation correction for ROI extraction. Xie and others, proposed Guided Filter based Single 
Scale Retinex (GFSSR) for finger vein image enhancement. Then Kauba and others[6,18,19] used a cascade of several preprocessing methods including (Image resize, LeeRegion, Normalization, CLAHE, circular Gabor filter, HFE filter and wavelet denoising) as an aim to improve the recognition performance using UTFVP database. They achieved different EER by cascading different methods of preprocessing and fusing the results of different feature extractors with different feature extractors including (LBP, RLT, MC and WLD). Shin and others ${ }^{[20]}$ used Lee mask for finger-vein boundary detection and empirical method based on finger-vein characteristics from the database. They used a combination of four directional Gabor filters to enhance thin vein and Retinex algorithm to enhance thick vein. However, the main drawback was in the time complexity for calculating the combination weights. Yahya and others ${ }^{[21]}$ presented a preprocessing scenario comprising of finger-vein boundary detection, rotation correction, binarization, thinning by morphological operations and ROI cropping. Sikarwar and Manmohan[22] use finger boundary edge detection followed by rotation correction and ROI extraction and finally enhancement by CLAHE method. Syarif and others ${ }^{[23]}$ used three main steps for preprocessing, finger center alignment, finger-vein boundary detection and image enhancement. They used pattern normalization model to solve the problem of misalignment, which is based on the hypothesis that the finger's cross-section is approximately ellipse and the vein that can be imaged is close to the finger surface. For finger-vein boundary detection, two Lee masks were used followed by tracking to remove the unwanted white pixels. For enhancement contrast adjustment and high pass filtering were used. Liu and others ${ }^{[24]}$ used image binarization then Sobel operator is applied to the entire image for edge map production which was subtracted from the binarized image. Finally, ROI extraction, alignment and enhancement were implemented. Liu and other's method seemed to be time consuming because of using two image threshold and the non-linear contract enhancement was done locally for overlapping $30 \times 30$ windows. Meng and others ${ }^{[25]}$ used Sobel operator to detect the edge of the finger then the width and height of the ROI was obtained by taking the maximum and minimum of $x$ and $y$ axes values of the finger profile followed by size normalization. Lu and others ${ }^{[26]}$ used Kirsch operator with eight directions for ROI extraction using UTFVP database.

Recently, Convolutional Neural Networks (CNN) have also been used for finger-vein recognition. In $\mathrm{CNN}$ the feature extraction is done inclusively, however the use of image preprocessing is optional. Hong and others ${ }^{[27]}$ used VGG Net-16 for finger-vein recognition using SDUMLA-HMT database. They pre-processed the image using ROI detection and resizing. Also Das and others ${ }^{[28]}$ used CNN for finger-vein identification using different types of public databases. The used preprocessing method of three steps, ROI detection using Lee masks, Image normalization for rotation correction and image enhancement using CLAHE technique. In general, the performance of $\mathrm{CNN}$ for finger-vein recognition is less than that of the hand-crafted feature methods. In addition, CNNs require to be retrained for database update, while for feature extraction based methods, the new subject only need to be added to the enrolled data.

\section{THE PROPOSE METHOD OF PREPROCESSING}

In this paper, a new scheme of preprocessing methods is introduced. It is based on a combination of previous and newly modified algorithms. The scheme as shown in figure (2) includes three major steps, RGB to Gray 
conversion, ROI localization and alignment and ROI enhancement. Another tuning alignment of ROI is done during the matching stage by the Phase Only Correlation (POC).The details of these steps are given in sections 4, 5 and 6.

\section{RGB TO GRAY CONVERSION}

Finger-vein images that are captured by most of the acquisition devices and provided by some of the public databases are RGB images with 24 bpp. In order to reduce the processing time and the complexity of calculations, it is necessary first to convert the images from RGB color image with 24 bpp to gray image with 8

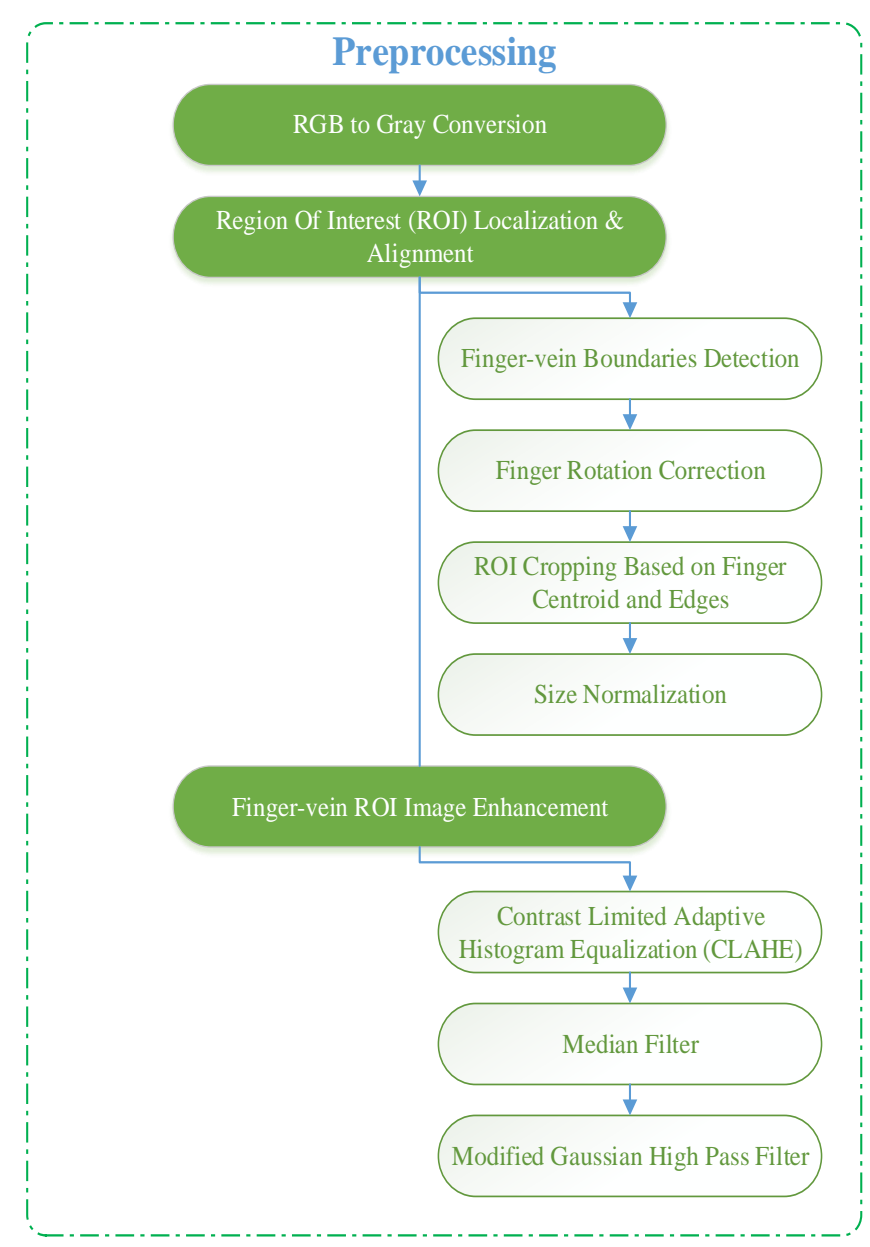

Fig. 2. The block-diagram of the proposed scheme of preprocessing

bpp. In this paper, the luminosity equation is used to convert RGB image to Gray image ${ }^{[29]}$ as shown below:

1. Gray $=R \times 0.299+G \times 0.588+B \times 0.114$
2. Where R, G and B denote Red, Green and Blue intensity values respectively.

\section{FINGER-VEIN LOCALIZATION AND ALIGNMENT}

In order to achieve good recognition accuracy, the region of interest of the finger-vein, usually called as ROI, must be extracted and aligned then enhanced before the stage of feature extraction. The process of extracting ROI involves the detection of the upper and lower borders of the finger and the alignment of the finger with $x$-axis to overcome the problem of finger displacement. The details of finger-vein detection and alignment are given in the following subsections.

\subsection{Finger-vein Boundary Detection}

Edge detection techniques are usually used to detect the finger-vein borders, specifically the upper and lower borders. As mentioned, various types of edge detectors have been used so far. The most common of them are the Lee mask used by [8], Sobel and Prewitt operators used by [13] and Hough transform used by [14-16]. These edge detectors are considered as time consuming since the size of Lee mask $4 \times 20$ is relatively large, while Prewitt and Sobel operators usually are performed in two rounds of convolution, whereas Hough transform is well known as a time consuming technique. In order to speed up the process of finger-vein boundary detection, in this paper two masks each of size $4 \times 6$ are used instead of two Lee masks each of size $4 \times 20$ and each convolution is performed only on one half of the image. The two masks are shown in figure (3)

\begin{tabular}{|r|r|r|r|r|r|}
\hline 1 & 1 & 1 & 1 & 1 & 1 \\
\hline 1 & 1 & 1 & 1 & 1 & 1 \\
\hline-1 & -1 & -1 & -1 & -1 & -1 \\
\hline-1 & -1 & -1 & -1 & -1 & -1 \\
\hline
\end{tabular}

(a)

\begin{tabular}{|c|c|c|c|c|c|}
\hline-1 & -1 & -1 & -1 & -1 & -1 \\
\hline-1 & -1 & -1 & -1 & -1 & -1 \\
\hline 1 & 1 & 1 & 1 & 1 & 1 \\
\hline 1 & 1 & 1 & 1 & 1 & 1 \\
\hline
\end{tabular}

(b)
Fig. 3. The two edge detection masks used in this paper (a) Mask for the upper half, (b) Mask for the lower half. The application of these two masks to samples of 
UTFVP and SDUMLA-HMT database are shown in figure (4).

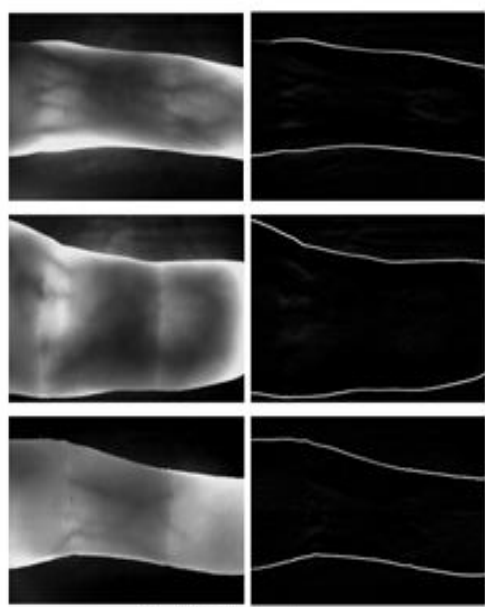

UTFVP Database
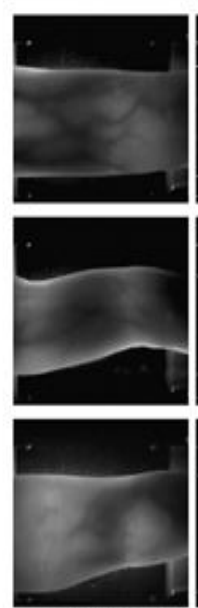

SDUMLA-HMT Database
Fig. 4. Samples of original and processed images for UTFVP and SDUMLA-HMT databases using the proposed masks, a and $b$ of figure 3 .

\subsection{Rotation Correction}

The displacement of the finger on the image acquisition device could result in finger rotation in $x-y$ plane. In order to resolve this problem, the central line between the upper and lower borders, which represents the finger base line, is localized. This is done by locating the midpoints on the $y$-axis for each $x$-value then a least square method is used to estimate the finger base line. Figure (5) illustrate the process of central line estimation.

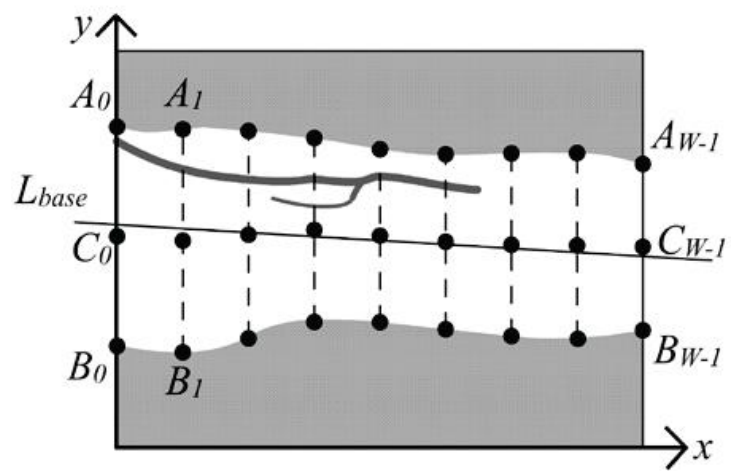

Fig. 5. Finger base line localization[30]

The finger upper and lower boundaries are represented by two sets of points $\left\{A_{\mathrm{x}}\right\}$ and $\left\{\mathrm{B}_{\mathrm{x}}\right\}$, where $x=0$ to $w-1$ and $w$ is the width of the image. The midpoints of the line segments $\left\{\overline{\mathrm{A}_{\mathrm{x}} \mathrm{B}_{\mathrm{x}}}\right\}$ are calculated and represented as a set of points $\left\{\mathrm{C}_{\mathrm{x}}\right\}$. Then a straight line know as the baseline is estimated to fit $\left\{\mathrm{C}_{\mathrm{x}}\right\}$ by the use of least square method[30,31]. Finally the rotation angle between the estimated base line and the horizontal direction is calculated and the affine transform for correcting the finger displacement is calculated.

The main procedures of rotation correction are:

i. Apply least mean square method to estimate the base-line from the point set $\left\{\mathrm{C}_{\mathrm{x}}\right\}$ using the line equation model as below:

$$
y=m x+c
$$

ii. Calculate $m$ and $c$ and the angle of rotation $\theta$ using equation (2)

$$
\theta=\arctan (m)
$$

iii. Construct affine transform from the multiplication of translation, scaling and rotation matrices.

Figure (6) shows the application of rotation correction to samples of UTFVP and SDUMLA-HMT databases. In this figure, the finger-vein image of UTFVP database is rotated counter clockwise, whereas the finger-vein image of SDUMLA-HMT database is rotated clockwise.

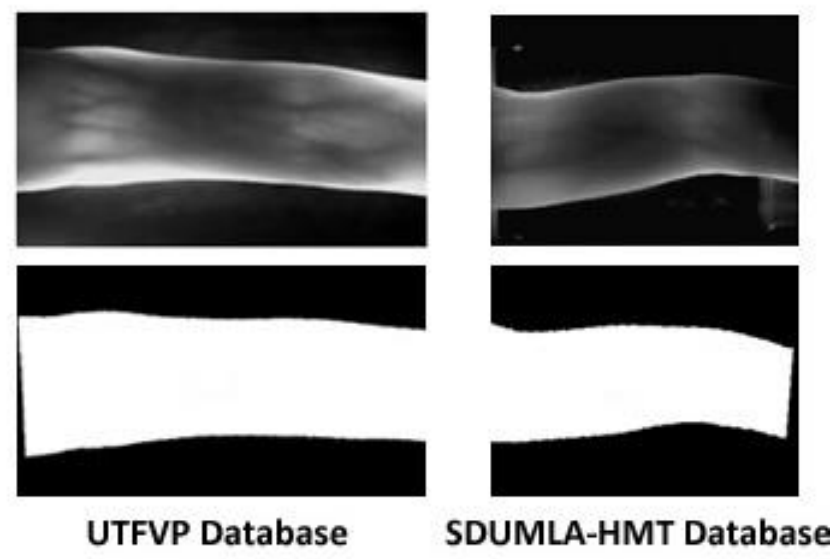

Fig. 6. Rotated samples of UTFVP and SDUMLA-HMT databases

\subsection{ROI Cropping}

The suggested method of ROI cropping is based on the 
central point of the finger-vein region. However, prior to determine this point, the removal of the unwanted white blocks that may appear in some databases such as SDUMLA-HMT after the process of rotation correction, will be necessary, otherwise the determination of the central point will not be accurate.

Here, these white blocks are removed by taking a sector of the finger border for each half. Then for each half, the average of the $y$ values for all the points on the sector is calculated and compared to the average of the $y$-values of all the points on the border and the points whose $y$ value exceeds the average by some threshold are removed. Figure (7) shows two samples of SDUMLAHMT images with white block region before and after the switch-of-points method. In this figure, the fingervein region is shown as white region and these white blocks are evident at both sides of the finger-vein.

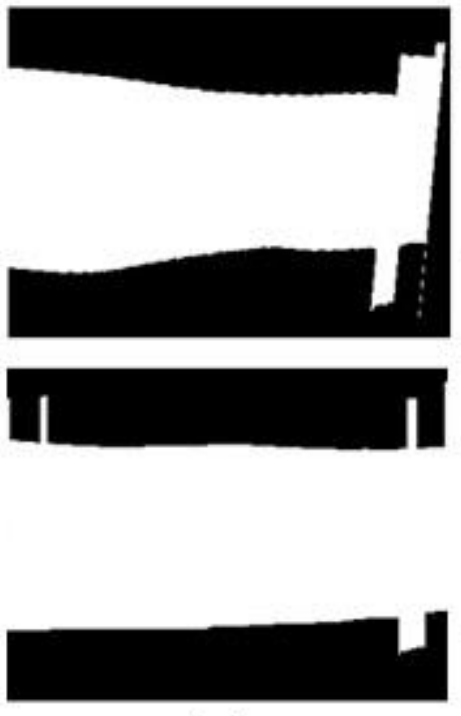

(a)
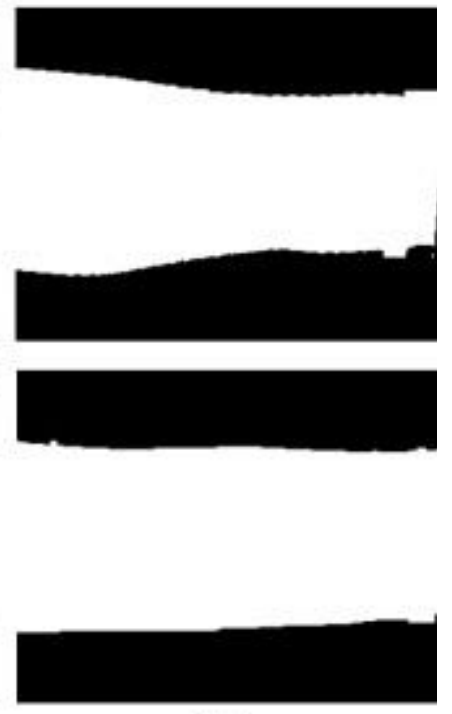

(b)
Fig. 7. (a) Image with unwanted white blocks (b) Image after removing white blocks

After the removal of the unwanted white blocks, ROI cropping is done by detecting the center of the finger region in the image, i.e., the center of the white region. To this end, the first invariant moment which represents the center of the finger region is calculated from the first and second moments by the use of the following equations ${ }^{[32] \text { : }}$

$$
\begin{aligned}
& M_{i j}=\sum_{x} \sum_{y} x^{i} y^{j} I(x, y) \\
& \text { Centroid: }\{\bar{x}, \bar{y}\}=\left\{\frac{M_{10}}{M_{00}}, \frac{M_{01}}{M_{00}}\right\}
\end{aligned}
$$

The ROI rectangle is localized using the extensions of upper and lower boundaries and the center of the finger region as follows:

i. The width of the ROI rectangle is specified by taking $80 \%$ of the total image width, i.e., by taking $40 \%$ from the left and right sides of the center point.

ii. The height of ROI rectangle is specified by the two horizontal lines passing through the lowest and highest index point.

Figure (8) shows the procedures of ROI cropping for different samples of UTFVP and SDUMLA-HMT database.
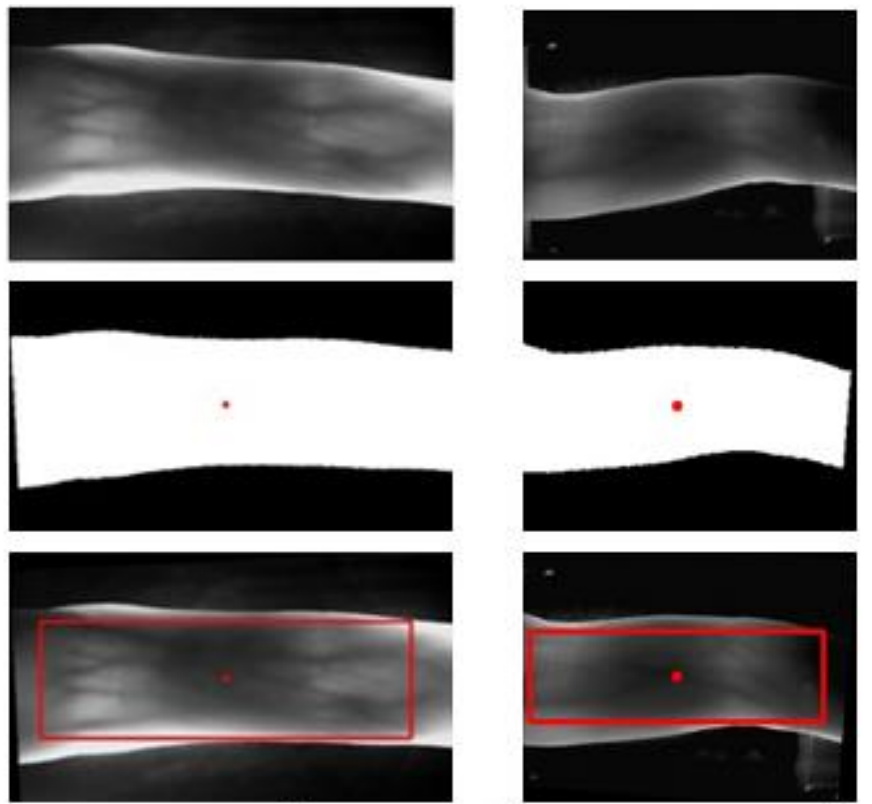

UTFVP Database

SDUMLA-HMT Database

Fig. 8. ROI rotation and cropping for two samples of UTFVP and SDUMLA-HMT databases

\subsection{Size Normalization}

The size of the cropped ROI is expected to be different 
from image to image as a result of the variations in finger-vein size and position on the acquisition devices. Therefore, size normalization is necessary to overcome the scale variant problem and at the same time to speed up the system. To this end, in this paper the size of all the extracted ROIs are unified to $192 \times 64$.

\section{ROI ENHANCEMENT}

The enhancement of the extracted ROI is a necessary step prior to feature extraction. Most of the previous methods achieved good results when Contrast Limited Adaptive Histogram Equalization (CLAHE) and/or image filtering are used for the enhancement. In this paper, CLAHE and two filters are used for ROI enhancement. The following subsection provides the details of these methods.

\subsection{Contrast Limited Adaptive Histogram Equalization (CLAHE)}

The images of the finger-veins are not always of high quality due to changes in illuminations, finger tissues and bones. Therefore, an effective method of image enhancement is required to improve the quality of the image and also to highlight the pattern of the finger-vein network to be ready for feature extraction. The method of CLAHE has been utilized successfully in previous works by ${ }^{[15,16]}$. In order to reveal more image details, in CLAHE method, the ROI is first divided into nonoverlapped windows then histogram equalization is performed for each window.

\subsection{Median Filter}

The technique of CLAHE sometimes enhances the contrast of the image in an exaggerated way resulting in noisy edges similar to salt-pepper noise. In order to reduce this noise a median filter of size $3 \times 3$ is used. In this case, Median filter will not affect the veins since they are a pattern of lines connected together with joints. Figure (9) shows the result of applying both CLAHE and
Median filter on samples of UTFVP and SDUMLA-HMT databases.
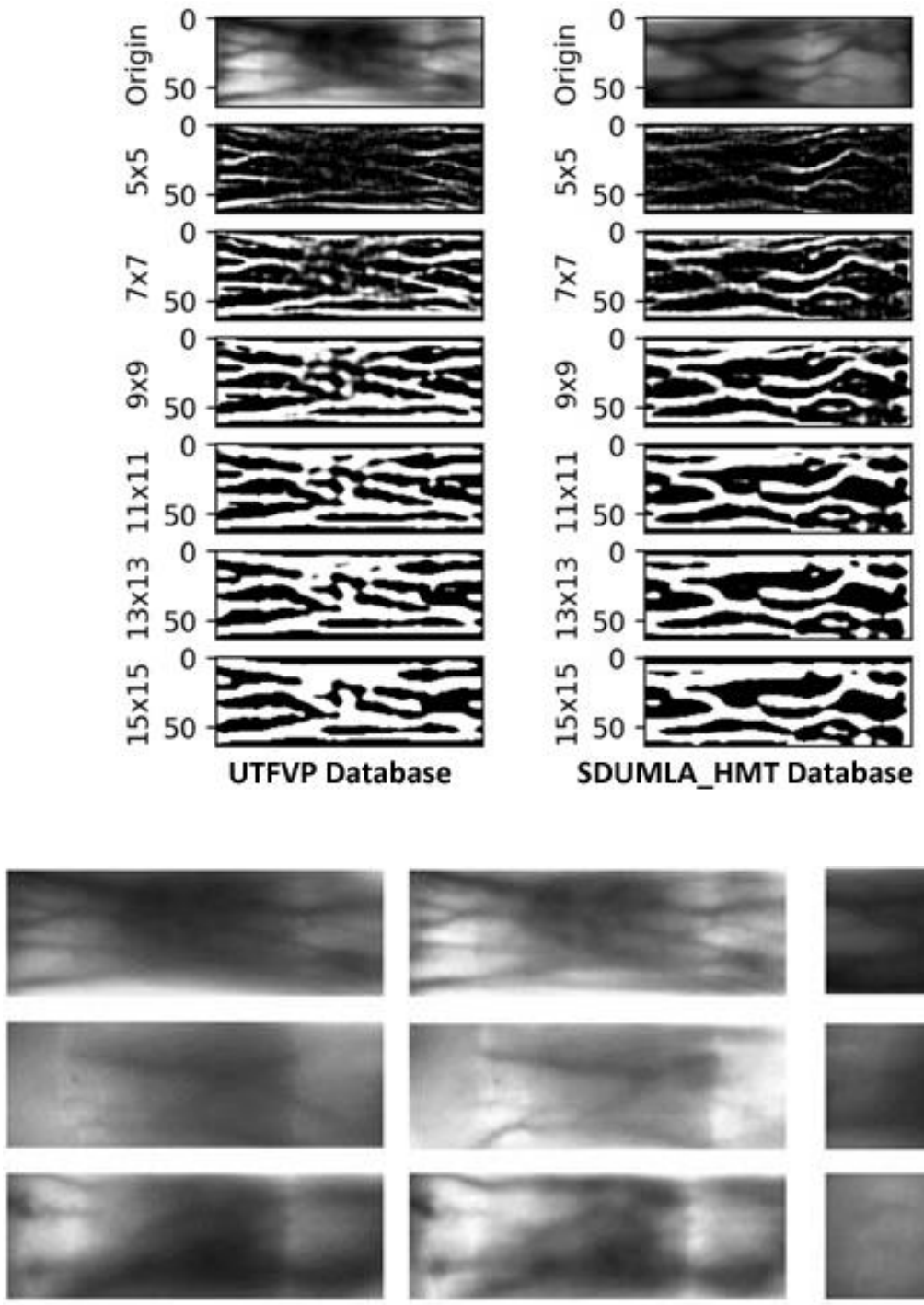

UTFVP Database

Fig. 9. The results of applying CLAHE and median filter for three samples of UTFVP and SDUMLA-HMT Databases

\subsection{Modified Gaussian High Pass Filter}

One of the most important steps to be taken prior to feature extraction is to make the finger-vein as clear as possible. Previously, Gabor filter is used intensively to enhance the veins ${ }^{[12,20,33]}$. However, determining the most appropriate frequencies and directions of the veins by the Gabor filter requires several implementations of 
Gabor filter and becomes a time complex process because the widths and directions of the veins vary from one to another. Gabor filters are traditionally used to obtain features of mid and high frequencies such as the patterns of iris. On the other hand, The modified Gaussian high pass filter has the advantage of obtaining low and mid frequencies such as the finger-veins and because it is symmetrical, features in all directions are enhanced ${ }^{[34]}$.

The modified Gaussian high passs filter is implemented using the following equation ${ }^{34]}$.

$$
H(x, y)=a\left(1-e^{\frac{-D^{2}(x, y)}{2 D_{0}^{2}}}\right)+b
$$

$D(x, y)$ Is calculated using the following equation:

$$
D(x, y)=\left[\left(x-x_{0}\right)^{2}+\left(y-y_{0}\right)^{2}\right]^{1 / 2}
$$

Where, $x_{0}$ and $y_{0}$ are the center of the Gaussian window, $\mathrm{D}(\mathrm{x}, \mathrm{y})$ is the distance from $\mathrm{x}, \mathrm{y}$ to $\mathrm{x}_{0}, \mathrm{y}_{0}$ and $\mathrm{D}_{0}$ is the standard deviation which specifyies the sharpness of the Gaussian function. Figure (10) shows the results of applying the modified Gaussian high pass filters of different size on ROI samples of UTFVP and SDUMLAHMT databases.

Fig. 10. The results of applying the modified Gaussian high pass filters of different size on ROI samples of UTFVP and SDUMLA-HMT databases.

\section{Database}

Several types of finger-vein databases which have been taken by devices of variant properties are available and are being used as standard dataset for evaluating the performance of finger-vein recognition systems. The most common examples of these databases are; UTFVP, SDUMLA-HMT, VERA and MMCBNU_6000[35-37]. In this work, UTFVP and SDUMLA-HMT are used for evaluating the preprocessing methods. The details of these two datasets are shown in table (1).

TABLE 1
Properties of UTFVP and SDUMLA-HMT Databases

\begin{tabular}{cccccc}
\hline \hline Database & $\begin{array}{c}\text { No. of } \\
\text { Subjects }\end{array}$ & $\begin{array}{c}\text { No. of } \\
\text { images }\end{array}$ & $\begin{array}{c}\text { No. of } \\
\text { finger/Subject }\end{array}$ & $\begin{array}{c}\text { No. of } \\
\text { Images/finger }\end{array}$ & $\begin{array}{c}\text { Image } \\
\text { size }\end{array}$ \\
\hline UTFVP & 60 & 1440 & $\begin{array}{c}6 \text { for both } \\
\text { hands } \\
\text { (middle, index, } \\
\text { ring) }\end{array}$ & $472 \times$ \\
SDUMLA- & 106 & 3816 & $\begin{array}{c}\text { 6 for both } \\
\text { hands } \\
\text { HMT }\end{array}$ & & \multicolumn{3}{c}{$\begin{array}{c}380 \\
\text { (middle, index, } \\
\text { ring) }\end{array}$} \\
\hline \hline
\end{tabular}

\section{RESULTS AND COMPARISONS}

The proposed scheme of preprocessing methods is applied to two finger-vein databases, UTFVP and SDUMLA-HMT. In order to reveal the efficiency of the preprocessing methods, the system is implemented in two modes (identification and verification using three different methods of feature extraction, Local Binary Pattern (LBP), Local Directional Pattern (LDP) and Local Line Binary Patter (LLBP). In order to assure the alignment of ROI for all the finger-vein images, POC is used during the matching stage. To achieve the optimum performance for each feature extraction, the system is implemented using Gaussian high pass masks of different size. For LLBP, in addition to different Gaussian mask size, different lengths of horizontal and vertical components are considered. The optimum performances for identification and verification modes for the three feature extractor are taken. The process of matching is implemented by the use of hamming distance measure. Figure (11) shows the Receiver Operating characteristic (ROC) curve for the three feature extraction methods. 


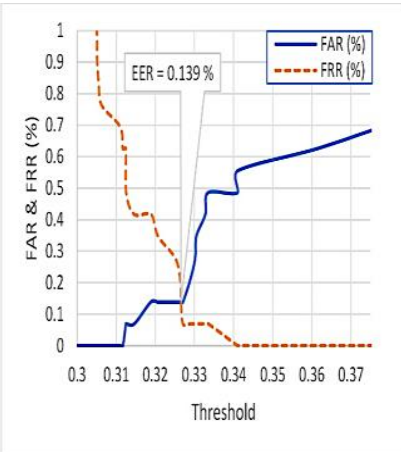

LBP

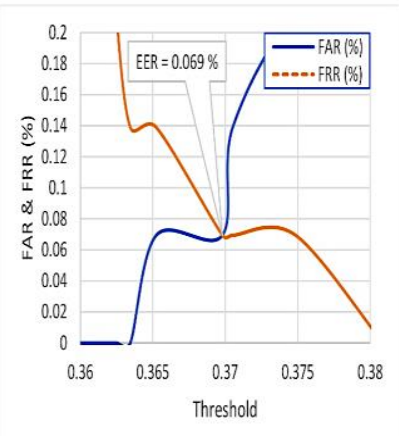

LDP

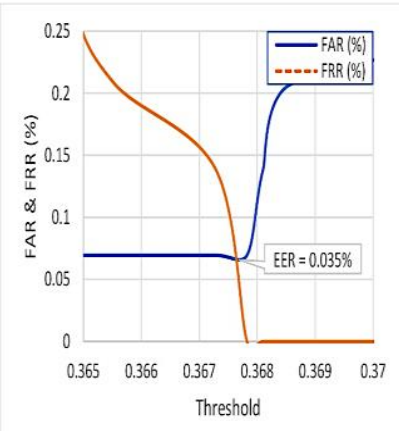

LLBP

UTFVP Database

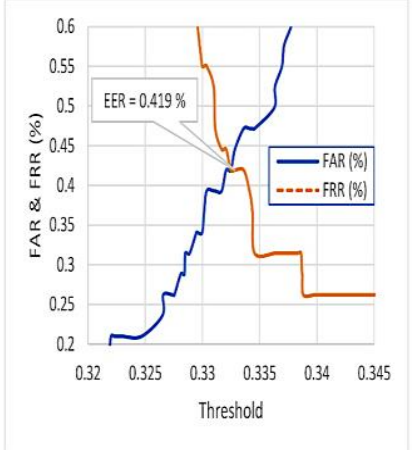

LBP

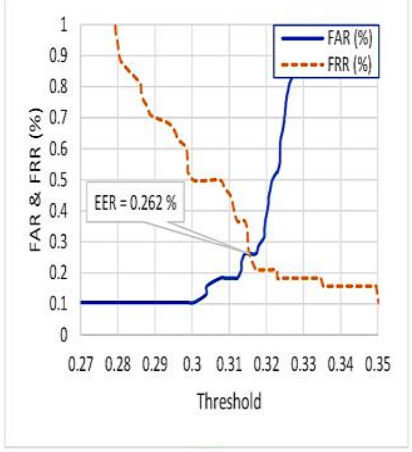

LDP

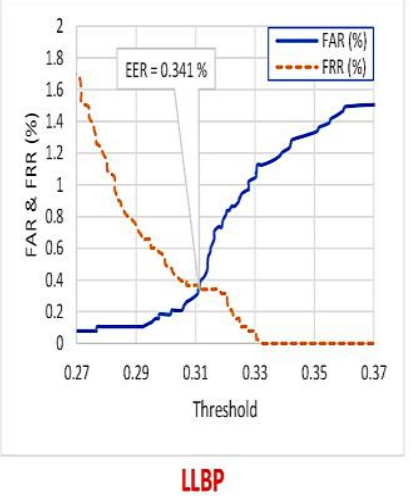

SDUMLA-HMT Database

Fig. 11. The Receiver Operating Characteristic (ROC) of the System for the three feature extractors and for the two databases, UTFVP and SDUMLA-HMT

Table (2) shows the performance measures including the Identification Recognition Rates (IRR) for identification mode and the Equal Error Rates (EER) for the verification mode for both datasets.

TABLE 2

Performance measures of finger-vein recognition system using the proposed scheme of preprocessing with different

feature extractors for UTFVP and SDUMLA-HMT databases.

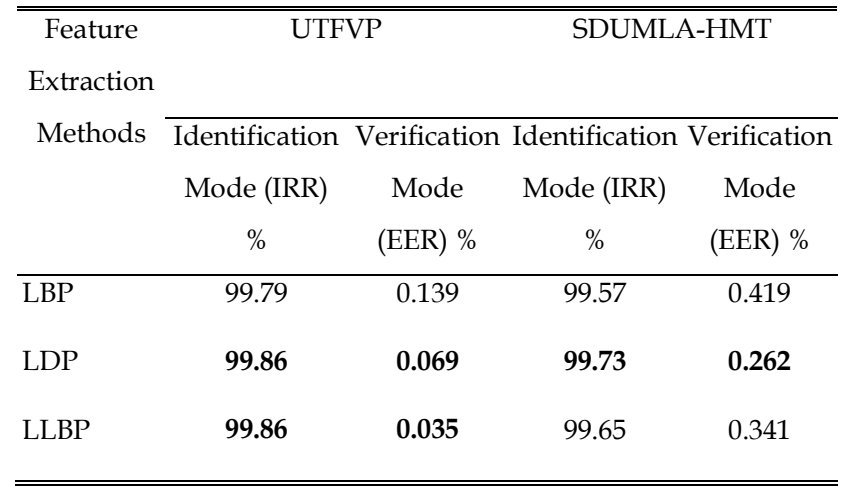

For UTFVP dataset, the IRR values of the identification mode for the three feature extractors are $(99.79 \%, 99.86 \%$ and $99.86 \%$ ) for LBP, LDP and LLBP respectively. The ERR values of the verification mode are $(0.139 \%, 0.069 \%$ and $0.035 \%$ ) for LBP, LDP and LLBP respectively. For SDUMLA-HMT dataset, the IRR values of the identification mode for the three feature extractors are (99.57\%, 99.73\% and 99.65\%) for LBP, LDP and LLBP respectively. These values of IRR are lower than those achieved by UTFVP database because the quality of SDUMLA-HMT images are worst than those of UTFVP. The ERR values of the verification mode are $(0.419 \%$, $0.262 \%$ and $0.341 \%$ ) for LBP, LDP and LLBP respectively. These results indicate that the proposed scheme of preprocessing achieved good performance with both types of databases and for modes, verification and identification.

In order to reveal the efficiency of the proposed method of preprocessing, the performances of finger-vein systems using previous methods of preprocessing with the same three feature extractors and for the similar datasets are shown in tables ( 3 and 4 ) for UTFVP and SDUMLA-HMT datasets. However, most of the previous works were restricted to one feature extractor and one mode, except the works done by ${ }^{[22,24]}$ for SDUMLA-HMT dataset.

According to this table, the IRR and EER values for the 
three feature extractors that are achieved by the proposed method of preprocessing are better than those achieved by previous methods of preprocessing for the same feature extractors.

TABLE 3

The performance comparison between the proposed and the previous methods of Preprocessing for three different feature extractors using UTFVP Database

\begin{tabular}{lcccccc}
\hline \multicolumn{1}{c}{$\begin{array}{c}\text { Preprocessing } \\
\text { Methods }\end{array}$} & \multicolumn{2}{c}{ LBP } & \multicolumn{2}{c}{ LDP } & \multicolumn{2}{c}{ LLBP } \\
\cline { 2 - 7 }$\quad$ Ipaper] & $\%$ & $\%$ & $\%$ & $\%$ & $\%$ & $\%$ \\
\hline Kauba and & - & 6.043 & - & - & - & - \\
others[6] & & & & & & \\
Kauba and & - & 6.0 & - & - & - & - \\
others[18] & & & & & & \\
Xie and others[17] & - & 5.70 & - & - & - & - \\
Lu and others[26] & - & 7.75 & - & 10.66 & - & - \\
Proposed method & 99.79 & 0.139 & 99.86 & 0.069 & 99.86 & 0.035 \\
\hline \hline
\end{tabular}

TABLE 4

The performance comparison between the proposed and the previous methods of Preprocessing for three different feature extractors using SDUMLA-HMT Database

The computation times for implementing the preprocessing steps are shown in Table (5). This time is subject to a computer with properties (Intel(R) Core(TM) i7-6700HQ CPU @ $2.60 \mathrm{GHz}$ ). The total time of (80) ms is reasonable. However, it is not wise to compare this time with the computation time of previous preprocessing method because of the difference between the properties of the computers used to implement the proposed preprocessing methods and the properties of the computers used to implement the previous preprocessing methods.

\section{TABLE 5}

The computation time of the preprocessing methods

$$
\text { Preprocessing Steps Time }
$$$$
\text { ms }
$$

\begin{tabular}{lc}
\hline Finger-vein boundary detection & 5 \\
Rotation correction & 25 \\
ROI cropping & 10 \\
ROI enhancement & 40 \\
Total Time & $\mathbf{8 0}$ \\
\hline \hline
\end{tabular}

\section{CONCLUSIONS}

The given scheme of preprocessing was shown to be efficient as the Identification Recognition Rates (IRRs) were high and the equal Error Rates (EERs) were low when used with different methods of feature extraction. The use of $4 \times 6$ masks instead of $4 \times 20$ has speeded up the process of finger-vein boundary detection. The use of the modified Gaussian high pass filter was effective for making the detection of the features more easy for the feature extractors. However, the size of the Gaussian high pass filter revealed little effect on the system performance. In addition, the given scheme of preprocessing has shown good efficiency for both fingervein databases, UTFVP and SDUMLA-HMT, despite the images of these databases are of different quality

\begin{tabular}{lcccccc}
\hline \multicolumn{1}{c}{$\begin{array}{c}\text { Preprocessing } \\
\text { Methods }\end{array}$} & \multicolumn{2}{c}{ LBP } & \multicolumn{2}{c}{ LDP } & \multicolumn{2}{c}{ LLBP } \\
\cline { 2 - 7 } [Paper] & IRR & EER & IRR & EER & IRR & EER \\
\hline Lu and others[16] & 98.48 & - & - & - & 98.56 & - \\
Shin and others[20] & - & 3.085 & - & - & - & - \\
Sikarwar and & & & 86.16 & 14.30 & & \\
Manmohan [22] & & & & & & \\
Liu and others[24] & & 10.2 & & 22.8 & & 10.9 \\
Proposed method & 99.57 & 0.419 & 99.73 & 0.262 & 99.65 & 0.341
\end{tabular}
because they were taken by different devices. The processing time for implementing the preprocessing scheme for all methods was reasonably good.

\section{Acknowledgements}

- This research work was implemented at the Computer Science Department / College of Science 
/ University of Duhok as a part of the Master degree requirements, 2017-2019.

- The authors would like to express their sincere gratitude to the University of Duhok and the College of Science for their continuous support to make this work possible.

\section{REFERENCES}

1. Yang, L., Yang, G., Wang, K., Liu, H., Xi, X., and Yin, Y., 2019, "Point Grouping Method for Finger Vein Recognition", IEEE Acess, doi: 10.1109/ACCESS.2019.2901017.

2. Liu, Z. and Song, S., 2012, "An embedded real-time fingervein recognition system for mobile devices", IEEE Transactions on Consumer Electronics. doi: 10.1109/TCE.2012.6227456.

3. Yang, J., Wei, J., and Shi, Y., 2018, "Accurate ROI Localization and Hierarchical Hyper-sphere Model for Finger-vein Recognition", Neurocomputing, NEUCOM 19858, doi.org/10.1016/j.neucom.2018.02.098.

4. Wang, H., Du, M., Zhou, J., and Tao, L., 2019, “Weber Local Descriptors with Variable Curvature Gabor Filter for Finger Vein Recognition", IEEE Acess, doi: 10.1109/ACCESS.2019.2928472.

5. Wang, K. Q., KRISA, A. S., WU,X. Q., and ZHAO, Q. S., 2012 "Finger vein recognition using LBP variance with global matching", Proceedings of the 2012 International Conference on Wavelet Analysis and Pattern Recognition, Xian, July, Pp. (196-200), doi: 10.1109/ICWAPR.2012.6294778.

6. Kauba, C., Reissig, J. and Uhl, A., 2014, "Pre-processing cascades and fusion in finger vein recognition", In Proceedings of the International Conference of the Biometrics Special Interest Group (BIOSIG'14), Darmstadt, Germany, September, Pp. (99-110).

7. Shaheed, K., Liu, H., Yang, G., Qureshi, I., Gou, J., and Yin, Y., 2018, "A Systematic Review of Finger Vein Recognition Techniques", Information, Vol. 9, issue 9, Pp. (1-29), doi.org/10.3390/info9090213/.

8. Lee, E. C., Lee, H. C., and Park, K. R., 2009, “Finger-vein Recognition Using Minutia-Based Alignment and Local Binary Pattern-Based Feature Extraction", International Journal of Imaging Systems and Technology, Vol. 19, issue 3, Pp. (179-186).

9. Park, K. R., 2011, “Finger Vein Recognition By Combining Global And Local Features Based On SVM", Computing and Informatics, Vol. 30, No. 2, Pp. (295-309).

10. Rosdi, B. A., Chai, W. S., and Shahrel A. S., 2011, "Fingervein Recognition Using Local Line Binary Pattern", Sensors, Vol. 11, Pp. (11357-11371), doi.org/10.3390/s111211357.

11. Ito, K., Aoki, T., Nakajima, H., Kpbayashi, K., Higushi, T., 2008, "A Palmprint Recognition Algorithm Using Phase Only Correlation", IEICETRANS, FUNDAMENTALS, Vol. E91-A, No. 4, Pp. (1023-1030).

12. Park, Y. H., and Park, K. R., 2012, "Image Quality Enhancement Using the Direction and Thickness of Vein Lines for Finger-Vein Recognition", International Journal of Advanced Robotic Systems, Vol. 9, issue 4, Pp. (1-10).

13. Pang S., Yin Y., Yang G., Li Y., 2012, “ Rotation Invariant Finger Vein Recognition", In: Zheng WS., Sun Z., Wang Y., Chen X., Yuen P.C., Lai J. (eds) Biometric Recognition, CCBR 2012, Lecture Notes in Computer Science, Vol. 7701, Springer, Berlin, Heidelberg, Pp. (151-156), doi.org/10.1007/978-3-642-35136-5_19.

14. Peng, J., Li, Q., Wang, N., Abd El-Latif, A. a., Niu, X., 2013, "An Effective Preprocessing Method for Finger Vein Recognition", Fifth International Conference on Digital Image Processing (ICDIP 2013), edited by Yulin Wang, Xie Yi, Proc. of SPIE Vol. 8878, doi: 10.1117/12.2030689.

15. Lu, Y., Yoon, S., and Park, D. S., 2013. "Finger-vein Recognition Based on Matching Score-Level Fusion of Gabor Features", The Journal of Korean Institute of Communications and Information Sciences, doi.org/10.7840/kics.2013.38a.2.174.

16. Lu, Y., Xie, S. J., Yoon, S., and Park, D. S., 2013. “Finger Vein Identification Using Polydirectional Local Line Binary Pattern", 2013 International Conference on ICT Convergence (ICTC), Jeju, Pp. (61-65), doi: 10.1109/ICTC.2013.6675307.

17. Xie, S. J., Lu, Y., Yoon, S., Yang, J., and Park, D. S., 2015, "Intensity Variation Normalization for Finger Vein Recognition Using Guided Filter Based Singe Scale Retinex", Sensors, Vol. 15, Pp. (17089-17105), doi:10.3390/s150717089.

18. Kauba, C. and Uhl, A., 2015, "Sensor ageing impact on finger-vein recognition," International Conference on Biometrics (ICB), Phuket, 2015, Pp. (113-120), doi: 10.1109/ICB.2015.7139084.

19. Kauba, C., Piciucco, E., Maiorana, E., Campisi, P., and Uhl, A., 2016, "Advanced variants of feature level fusion for finger vein recognition," In 2016 International Conference of the Biometrics Special Interest Group (BIOSIG), Sept, Pp. (1-7).

20. Shin, K. Y., Park, Y. H., Nguyen, D. T., and Park K. R., 2014, “Finger-Vein Image Enhancement Using a FuzzyBased Fusion Method with Gabor and Retinex Filtering", Sensors, 14, Pp. (3095-3129), doi:10.3390/s140203095.

21. Yahaya, Y. H., Shamsuddin, S. M., and Leng, W. Y., 2016, "Finger Vein Feature Extraction Using Discretization", 4th International Conference on Artificial Intelligence and Computer Science, (AICS2016), 28 - 29 November, 
Langkawi, MALAYSIA.

22. Sikarwar, P., and Manmohan, "Finger vein recognition using local directional pattern," 2016 International Conference on Inventive Computation Technologies (ICICT), Coimbatore, 2016, Pp. (1-5), doi: 10.1109/INVENTIVE.2016.7823248.

23. Syarif, M.A., Ong, T.S., Teoh, A.B.J., and Tee, C., 2017, "Enhanced Maximum Curvature Descriptors for Fingervein Verification", Multimedia Tools and Applications, Vol.76, Issue 5, Pp. (6859-6887), doi.org/10.1007/s11042016-3315-4

24. Liu, H., Yang, L., Yang, G., and Yin, Y., 2018, "Discriminative Binary Descriptor for Finger Vein Recognition", In IEEE Access, vol. 6, Pp. (5795-5804), doi:10.1109/ACCESS.2017.2787543.

25. Meng, X., Xi, X., Yang, G., and Yin, Y., 2018, “Finger vein recognition based on deformation information", SCIENCE CHINA, Information Sciences, Vol. 61, doi.org/10.1007/s11432-016-9037-0.

26. Lu, Y., Yoon, s., Wu, S., and Park, D. S., 2018, "pyramid Histogram Of Double Competitive Pattern For Finger Vein Recognition", IEEE Acess, doi:10.1109/ACCESS.2018.2872493

27. Hong, H. G., Lee, M. B., and Park K. R., 2017, "Convolutional Neural Network-Based Finger-Vein Recognition Using NIR Image", Sensors, Vol. 17, doi.org/10.3390/s17061297.

28. Das, R., Piciucco, E. Maiorana and P. Campisi, 2019, "Convolutional Neural Network for Finger-Vein based Biometric Identification", in IEEE Transaction for Information Forensics and Security, vol. 14, no. 2, Pp. (360373).

29. Tallam R. R., Temgire, S. S., and Zirange, R. M., 2014, "Finger Vein Recognition System Using Image Processing", International Journal of Electrical, Electronics and Data Communication, Vol.-2, Issue-5, Pp. (64-68).

30. Huang, B., Dai, D., Li, R., Tang, D., and Li, W., 2010, "Finger-vein Authentication Based on Wide Line Detector and Pattern Normalization", 2010 International Conference on Pattern Recognition, Pp. (1269-1272).

31. Lu, Y., Xie, S. J., Yoon, S., Yang, J., and Park, D. S., 2013, "Robust Finger Vein ROI Localization Based on Flexible Segmentation", Sensors, Vol. 13, No. 11, Pp (14339-14366), doi: 10.3390/s131114339.

32. Huang, Z. , and Leng, J., 2010, “Analysis of Huâ€ $€^{\mathrm{TM}_{\mathrm{S}}}$ Moment Invariants on Image Scaling and Rotation", Proceedings of the 2nd International Conference on Computer Engineering and Technology (ICCET), Pp. (476480).

33. Kauba, C., Piciucco, E., Maiorana, E., Campisi, P., and Uhl, A., 2018, "Robustness of finger-vein recognition", HandBased Biometrics: Methods and Technology, Chapter nine, Pp. (193-216), doi: 10.1049/PBSE008E.

34. Lee, E. C., Jung, H., and Kim, D., 2011, “New Finger Biometric Method Using near Infrared Imaging", Sensors, Vol. 11, Pp. (2319-2333), doi.org/10.3390/s110302319.

35. Yin Y., Liu L., Sun X., 2011, "SDUMLA-HMT: A multimodal Biometric Database", In Biometric Recognition by (Sun, Z., L., J., Chen, X., Tan, T. (Eds.)), Springer Berlin Heidelberg, Pp. (260-268).

36. Lu, Y., Xie, S. J., Yoon, S., Wang, Z., and Park, D. S., 2013, "An Available Database for the Research of Finger Vein Recognition", 2013 6th International Congress on Image and Signal Processing (CISP 2013), Pp (410-415).

37. Vanoni, M., Tome, P., El Shafey, L., and Marcel, S., 2014, "Cross-Database Evaluation Using an Open Finger Vein Sensor", 2014 IEEE Workshop on Biometric Measurements and Systems for Security and Medical Applications (BIOMS) Proceedings, Rome, Pp. (30-35), doi: 10.1109/BIOMS.2014.6951532. 